\title{
Thermodynamics and phase transitions in the Born-Infeld-anti-de Sitter black holes
}

\author{
Yun Soo Myung ${ }^{1, a}$, Yong-Wan Kim ${ }^{1, b}$, and Young-Jai Park ${ }^{2, c}$ \\ ${ }^{1}$ Institute of Basic Science and School of Computer Aided Science, \\ Inje University, Gimhae 621-749, Korea \\ ${ }^{2}$ Department of Physics, Sogang University, Seoul 121-742, Korea
}

\begin{abstract}
We investigate the Born-Infeld-anti-de Sitter black hole (BIAdS) solutions in the four dimensions, which is a nonlinear generalization of the Reissner-Norström-AdS black hole (RNAdS). We analyze all thermodynamic quantities of the BIAdS in the canonical ensembles, which are characterized by the charge $Q$, the mass $M$, the nonlinear parameter $b$, comparing with those of the RNAdS and SchwarzschildAdS black hole. We find the forbidden region of $0 \leq b Q<0.5$ for the presence of a charged BIAdS. We also discuss the Hawking-Page phase transitions in the BIAdS black holes. Here we obtain a new Hawking-Page phase transition for the $b Q=0.5$ critical BIAdS.
\end{abstract}

PACS numbers: 04.70.Dy, 04.60.Kz.

Keywords: Born-Infeld-anti-de Sitter black hole; Thermodynamics; HawkingPage phase transition.

aysmyung@inje.ac.kr

${ }^{b}$ ywkim65@gmail.com

cyjpark@sogang.ac.kr 


\section{Introduction}

Since Born and Infeld (BI) proposed a nonlinear electrodynamics [1], BI action has received renewed attention in string theory $[2,3,4]$. For various motivations, extending the Reissner-Nordström black hole (RNAdS) in Einstein-Maxwell theory to the charged black hole solutions in Einstein-BornInfeld (EBI) theory has attracted much attention in recent years, for example, see $[5,6,7,8,9,10,11,12,13,14,15]$. In particular, Einstein gravity in $(2+1)$ dimensions $[16,17,18]$ has been intensively studied because of the existence of black holes solutions in $(2+1)$-dimensional anti-de Sitter (AdS) spacetimes [19, 20, 21, 22], which possess certain features inherent to the $(3+1)$-dimensional black holes. Recently, we have systematically obtained all thermodynamic quantities of the EBI black holes in three dimensions by comparing those of the Maxwell and BTZ black holes [23]. These are all between non-rotating uncharged black hole (NBTZ) and charged black hole (CBTZ) [24]. This result in 3D EBI black holes (BIBTZ) may provide new insights towards a better understanding of the $(3+1)$-dimensional BornInfeld-anti-de Sitter black holes (BIAdS), whose thermodynamic analysis is regarded as a nontrivial task to carry out completely $[13,14]$.

On the other hand, since the pioneering work of Hawking-Page on the phase transition between thermal AdS and AdS black hole in four dimensions [25], the research of the black hole thermodynamics has greatly improved additionally suggesting that there may exist a different phase transition between small and large black holes (HP1) in the RNAdS for a fixed charge $Q<Q_{c}[26,27,28]$ and AdS Gauss-Bonnet black holes [29, 30, 31, 32]. In contrast, in the conventional Hawking-Page phase transition (HP2), one generally starts with thermal radiation in AdS space appearing an unstable small black hole with negative heat capacity. Then, since the heat capacity changes from negative infinity to positive infinity at the minimum temperature, the large black hole with positive heat capacity finally comes out as a stable object. There is a change of the dominance at the critical temperature: from thermal radiation to black hole [25].

In this paper, we address these issues for the BIAdS, which is a nonlinear generalization of the RNAdS. We completely analyze all thermodynamic quantities of the BIAdS in the canonical ensemble, which is characterized by charge $Q$, mass $M$, and the nonlinear parameter $b$. Note that the work of the BIAdS in the grand canonical ensemble was carried out in Ref. [14]. Moreover, we obtain a new type of the Hawking-Page phase transition (HP3) 
in the BIAdS black holes, which has been still not observed in the previous works [25, 26, 27, 28, 29, 30, 33, 34, 35].

The organization of this work is as follows. In Sec. 2, we carefully analyze the possible BIAdS black hole solutions, which are classified by the charge $Q$ and the nonlinear parameter $b$. In Sec. 3, we investigate all thermodynamic properties of the allowed BIAdS as a nonlinear realization of the RNAdS. In Sec. 4, we present a new phase transition in the BIAdS black holes. We summarize and discuss our results in Sec. 5. In Appendix, we derive the free energy using the Euclidean action approach.

\section{Structure of BIAdS black holes}

Now, let us consider a $(3+1)$-dimensional gravity coupled with nonlinear electrodynamics known as the BIAdS action [13, 33, 34]

$$
S=\int d^{4} x \sqrt{-g}\left(\frac{R-2 \Lambda}{16 \pi G}+L(F)\right)
$$

where

$$
L(F)=\frac{b^{2}}{4 \pi G}\left(1-\sqrt{1+\frac{2 F}{b^{2}}}\right)
$$

with $F \equiv F^{\mu \nu} F_{\mu \nu} / 4$. Here, the constant $b$ is the Born-Infeld parameter, which is related to the string tension $\alpha^{\prime}$ as $b=1 /\left(2 \pi \alpha^{\prime}\right)$, and $\Lambda=-3 / l^{2}$ is the cosmological constant. Hereafter we choose $G=1$ for simplicity. Note that this Lagrangian reduces to the RNAdS one in the limit $b^{2} \rightarrow \infty$.

By solving the equations of motion for the gauge field $A_{\mu}$ and the gravitational field $g_{\mu \nu}$, the BIAdS black hole solutions $[13,33,34]$ can be written as

$$
d s^{2}=-f(r) d t^{2}+f(r)^{-1} d r^{2}+r^{2} d \Omega^{2} .
$$

Here, the metric function $f(r)$ is given by

$$
\begin{aligned}
f(r)= & 1-\frac{2 M}{r}+\frac{r^{2}}{l^{2}}+\frac{2 b^{2} r^{2}}{3}\left(1-\sqrt{1+\frac{Q^{2}}{b^{2} r^{4}}}\right) \\
& +\frac{4 Q^{2}}{3 r^{2}} \mathcal{F}\left(\frac{1}{4}, \frac{1}{2}, \frac{5}{4},-\frac{Q^{2}}{b^{2} r^{4}}\right),
\end{aligned}
$$

where $\mathcal{F}$ is a hypergeometric function [39], and only non-zero component with the electric charge $Q$ is given by $F^{01}=-E=-Q / \sqrt{r^{4}+Q^{2} / b^{2}}$. Hereafter we only consider $Q \geq 0$ and $b \geq 0$ without any loss of generality. At this stage 
we note two limiting cases as guided black holes to study the BIAdS black holes. In the limit $Q \rightarrow 0, f(r)$ reduces to the Schwarzschild-anti de Sitter black hole (SAdS) case, while in the limit $b \rightarrow \infty$ and $Q \neq 0, f(r)$ reduces to the RNAdS case. However, the BIAdS should have the main feature of the RNAdS: two horizons $r_{ \pm}$and extremal black hole because the BIAdS is a nonlinear generalization of the RNAdS. This implies that the SAdS with single horizon does not belong to the category of the RNAdS and thus it should be disconnected to the BIAdS.

On the other hand, the ADM mass $M$ defined by $f(r)=0$ is given by

$$
\begin{aligned}
M\left(r_{+}, Q, b\right)= & \frac{r_{+}}{2}+\frac{r_{+}^{3}}{2 l^{2}}+\frac{b^{2} r_{+}^{3}}{3}\left(1-\sqrt{1+\frac{Q^{2}}{b^{2} r_{+}^{4}}}\right) \\
& +\frac{2 Q^{2}}{3 r_{+}} \mathcal{F}\left(\frac{1}{4}, \frac{1}{2}, \frac{5}{4},-\frac{Q^{2}}{b^{2} r_{+}^{4}}\right)
\end{aligned}
$$

with the outer horizon $r=r_{+}$. In order to describe the BIAdS with $Q \neq 0$ and $b \neq 0$, which is the generalization of the RNAdS, we first have to know the extremal BIAdS. In this case, both $f(r)$ and $\frac{d f(r)}{d r}$ have to be zero at the degenerate horizon. Such conditions will lead to the equation

$$
1+\left(2 b^{2}+\frac{3}{l^{2}}\right) r_{e}^{2}-2 b^{2} \sqrt{r_{e}^{4}+\frac{Q^{2}}{b^{2}}}=0
$$

The solution is given by

$$
r_{e}^{2}=\frac{l^{2}}{6}\left(\frac{1+\frac{3}{2 b^{2} l^{2}}}{1+\frac{4}{4 b^{2} l^{2}}}\right)\left[-1+\sqrt{1+\frac{12\left(1+\frac{3}{4 b^{2} l^{2}}\right)}{b^{2} l^{2}\left(1+\frac{3}{2 b^{2} l^{2}}\right)^{2}}\left(b^{2} Q^{2}-\frac{1}{4}\right)}\right] .
$$

Then, we require the condition $b Q \geq 0.5$ in order to have a real root for $r_{e}^{2}$. Note that $r_{e}^{2}=0$ for $b Q=0.5$, while $r_{e}^{2}$ reduces to the RNAdS in the limit $b \rightarrow \infty$. On the other hand, for $0 \leq b Q<0.5$, we could not find the extremal BIAdS. Hence, we call this as the forbidden region for the BIAdS. As a result, we propose that the meaningful parameter space for the BIAdS is not the whole, but

$$
0.5 \leq b Q \leq \infty
$$

We call the lower bound as the critical BIAdS and the upper bound of $b \rightarrow \infty$ as the RNAdS. This lower bound could be understood from the fact that the presence of $b$ leads to the screening effects on the electric field $E$, and thus makes the role of charge less important. Hence, for $0 \leq b Q<0.5$, any charged black hole does not exist. 


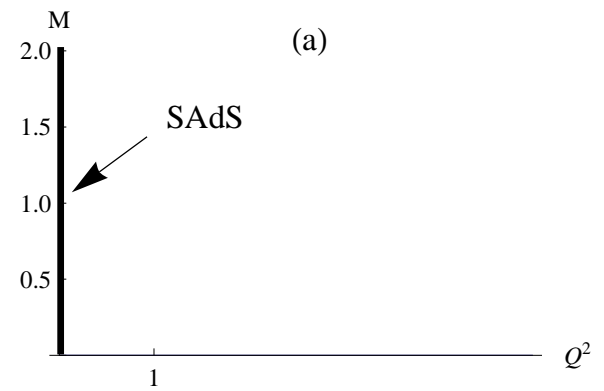

(c)

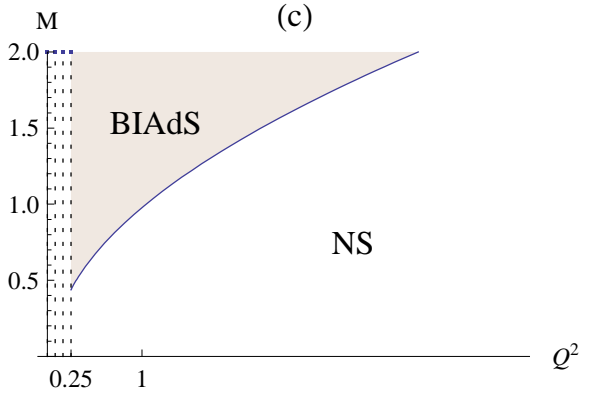

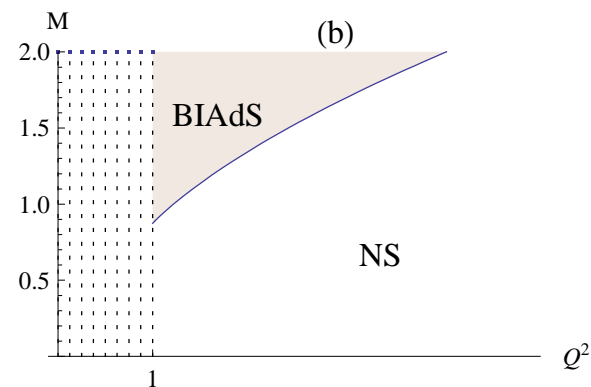

(d)

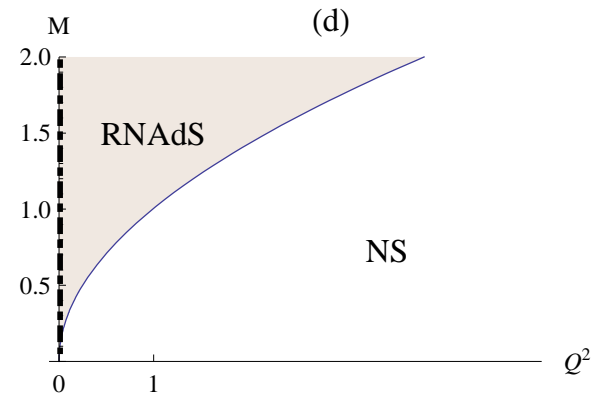

Figure 1: The mass function graphs $M$ vs $Q^{2}$ with $l=10$ : (a) the SAdS case $(Q=0)$, two BIAdS cases with (b) $b=0.5$ and (c) $b=1.0$, and (d) the RNAdS case, respectively. The naked singularity regions (NS) are white areas below solid curves of the extremal BIAdS, while the forbidden regions (FR) are dotted areas between positive $M$-axis $(Q=0)$ and $b Q=\frac{1}{2}$.

Next, for the case of $b Q \geq 0.5$, the mass of the extremal black hole is given by

$$
M_{e} \equiv M\left(r_{e}, Q, b\right),
$$

which reduces to the well-known RNAdS case in the limit of $b \rightarrow \infty$. As is shown in Fig. 1, when $b$ increases $(b=0.5 \rightarrow 1 \rightarrow \infty)$, the dotted forbidden regions $(\mathrm{FR})$ are getting narrow and narrow while naked singularity regions (NS) are increasing wide and wide.

Let us discuss the behavior of mass as a function of horizon radius $r=r_{ \pm}$. First, we consider the case of $b Q>0.5$. If $M>M_{e}$, then there will be two horizons: the inner $r_{-}$and outer $r_{+}$. For $M=M_{e}$ there will be a degenerate horizon of $r_{-}=r_{+}=r_{e}$. If $M<M_{e}$, there are no horizons as shown in Fig. 2 , and it will yield a naked singularity. Second, in the case of $b Q=0.5, r_{e}=0$ is the extremal point and for $M>M_{e}$, one has one horizon $r_{+}$only. Finally, there exists a dotted forbidden region described by $0 \leq b Q<0.5$ including the SAdS, which can not be allowed for the BIAdS having the limiting case of the RNAdS. This can be easily checked by expanding $M\left(r_{+}, Q, b\right)$ in series 


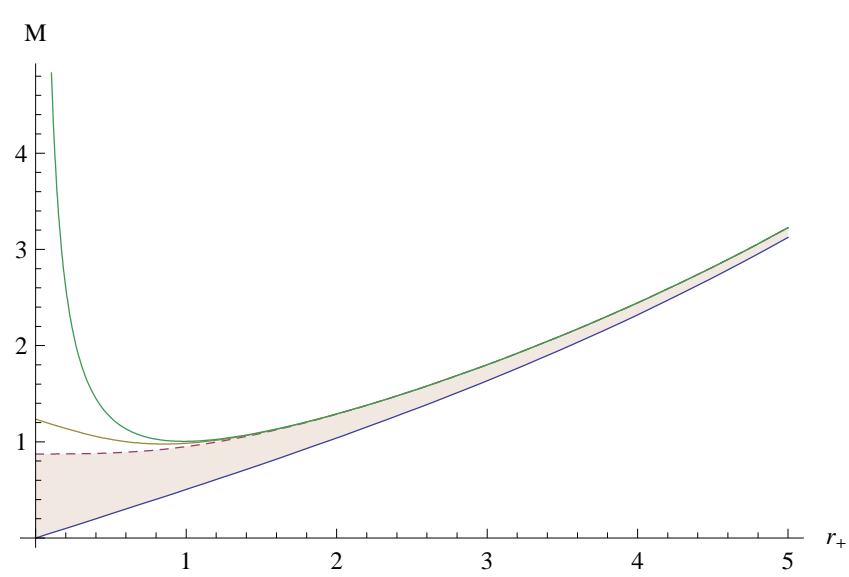

Figure 2: The mass function graphs $M$ vs $r_{+}$with $l=10$ from bottom to top: the SAdS case $(Q=0)$, two BIAdS cases $(Q=1)$ with $b=0.5$ and $b=1.0$, and the RNAdS case, respectively. The dotted line stands for $b=0.5$. The shaded region represents the forbidden region of BIAdS: $0 \leq b Q<0.5$.

near $r_{+}=0$ as

$$
M\left(r_{+}, Q, b\right) \approx M_{0}-\left(b Q-\frac{1}{2}\right) r_{+}+\left(\frac{b^{2}}{3}+\frac{1}{2 l^{2}}\right) r_{+}^{3}+\mathcal{O}\left[r_{+}\right]^{5}
$$

with

$$
M_{0}=\frac{\Gamma\left[\frac{1}{4}\right]^{2}}{6 \sqrt{\pi}} \sqrt{b Q^{3}} .
$$

This shows clearly that there exists a non-zero mass $M_{0}$ depending on $b, Q$ even at $r_{+}=0$. In fact, $M_{0}$ is hard to be interpreted as the mass of a black hole. Therefore, for the case of $0 \leq b Q<0.5$ (the positive slop of linear term), we could not obtain the BIAdS black hole solution having the extremal configuration. However, for the case of $b Q>0.5$, the slop of the linear term is negative, explaining how the extremal configurations could be being developed. For the case of $b Q=0.5$, the linear term disappears and its case is marginal.

\section{Thermodynamics of BIAdS black holes}

Now, we are ready to analyze the BIAdS black hole, which is really a nonlinear generalization of the RNAdS in the canonical ensembles. The Hawking 


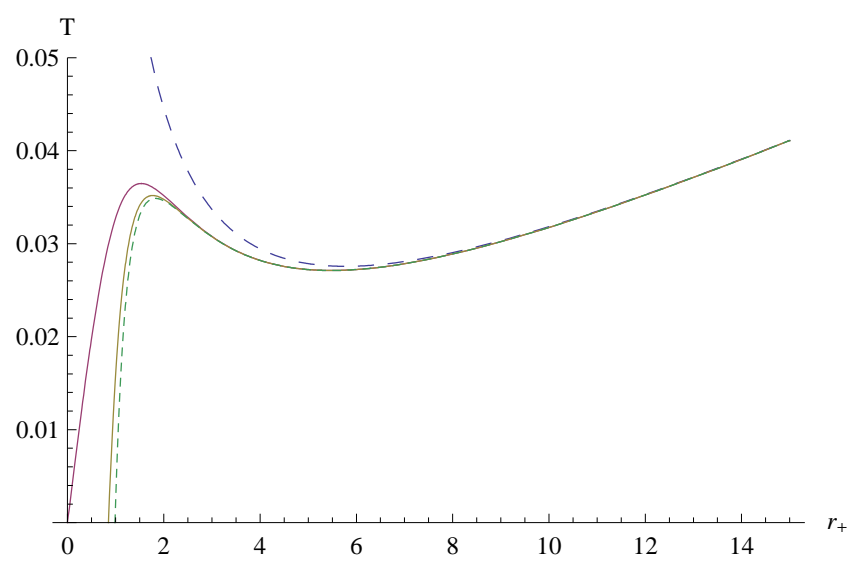

Figure 3: Temperature graphs $T$ vs $r_{+}$with $l=10$ : the dashed line is for the SAdS case $(Q=0)$, two BIAdS cases $(Q=1)$ with $b=0.5, b=1$, and the dotted line for the RNAdS case, respectively.

temperature defined by $T_{H}=f^{\prime}\left(r_{+}\right) / 4 \pi$ takes the form

$$
T_{H}\left(r_{+}, Q, b\right)=\frac{1}{4 \pi}\left(\frac{1}{r_{+}}+\frac{3 r_{+}}{l^{2}}+2 b^{2} r_{+}\left[1-\sqrt{1+\frac{Q^{2}}{b^{2} r_{+}^{4}}}\right]\right) .
$$

We note that in the limit $Q \rightarrow 0, T_{H}$ reduces to the SAdS case, while in the limit of $b \rightarrow \infty$ and $Q \neq 0, T_{H}$ reduces to the well-known RNAdS case. Then, using the Eqs. (5) and (12), the heat capacity $C\left(r_{+}, Q, b\right)=\left(d M / d T_{H}\right)_{Q}$ for a fixed-charge is obtained to be

$$
C=\frac{2 \pi r_{+}^{2} \sqrt{1+\frac{Q^{2}}{b^{2} r_{+}^{4}}}\left[3 r_{+}^{4}+l^{2}\left(r_{+}^{2}+2 b^{2}\left(1-\sqrt{1+\frac{Q^{2}}{b^{2} r_{+}^{4}}}\right) r_{+}^{4}\right)\right]}{3 \sqrt{1+\frac{Q^{2}}{b^{2} r_{+}^{4}}} r_{+}^{4}+l^{2}\left[-\sqrt{1+\frac{Q^{2}}{b^{2} r_{+}^{4}}} r_{+}^{2}+2 Q^{2}-2 b^{2}\left(1-\sqrt{1+\frac{Q^{2}}{b^{2} r_{+}^{4}}}\right) r_{+}^{4}\right]} .
$$

The proper on-shell free energy defined by $F\left(r_{+}, Q, b\right)=M-M_{e}-T_{H} S_{B H}$ with the Bekenstein-Hawking entropy $S_{B H}=\pi r_{+}^{2}$ is given by

$$
\begin{aligned}
F & =\frac{r_{+}}{4}-\frac{r_{+}^{3}}{4 l^{2}}-\frac{b^{2} r_{+}^{3}}{6}\left(1-\sqrt{1+\frac{Q^{2}}{b^{2} r_{+}^{4}}}\right) \\
& +\frac{2 Q^{2}}{3 r_{+}} \mathcal{F}\left(\frac{1}{4}, \frac{1}{2}, \frac{5}{4},-\frac{Q^{2}}{b^{2} r_{+}^{4}}\right)-M_{e} .
\end{aligned}
$$

Here, we choose the extremal black hole as background because we are working with the fixed-charge $Q$ ensemble [26]. In order to prove the correctness of 

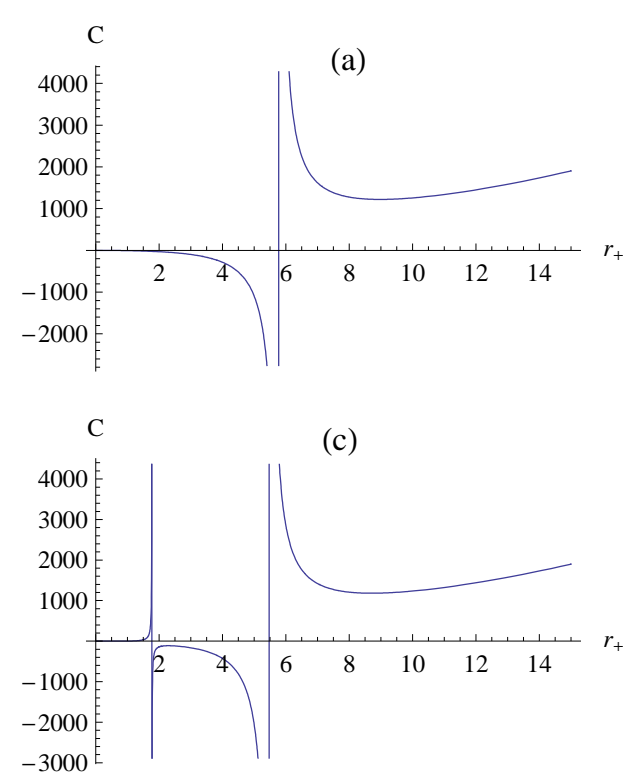
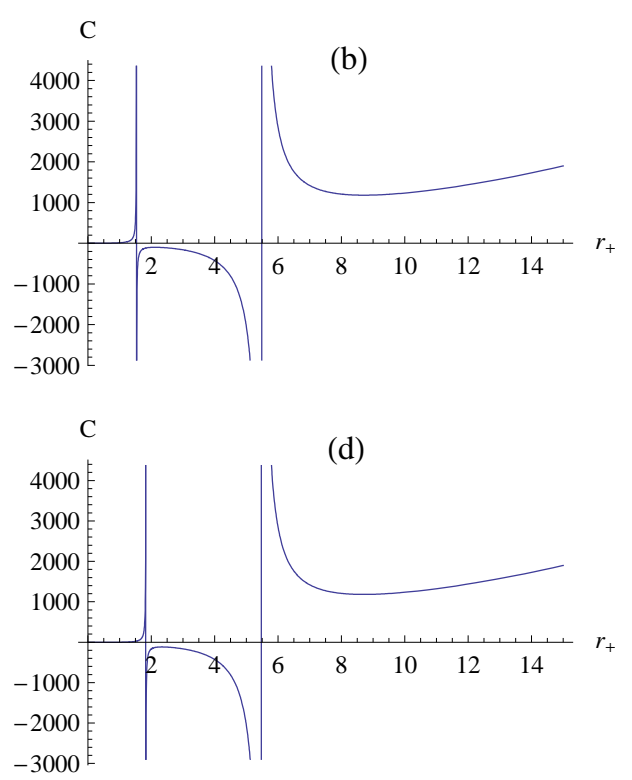

Figure 4: Specific heat graphs $C$ vs $r_{+}$with $l=10$ : (a) the SAdS case $(Q=0)$, two BIAdS cases $(Q=1)$ with (b) $b=0.5$, (c) $b=1.0$, and (d) the RNAdS case, respectively.

free energy, $F$ will be derived from the Euclidean action approach in the Appendix. Also, the free energy (14) may be obtained using the 2-dimensional dilaton gravity through the dimensional reduction [36, 37, 38, 23].

Note that in the limit $Q \rightarrow 0$, the heat capacity $C$ and free energy $F$ reduce to the SAdS case as

$$
\begin{aligned}
& C^{S A d S}\left(r_{+}\right)=2 \pi r_{+}^{2}\left[\frac{3 r_{+}^{4}+l^{2} r_{+}^{2}}{3 r_{+}^{4}-l^{2} r_{+}^{2}}\right], \\
& F^{S A d S}\left(r_{+}\right)=\frac{r_{+}}{4}\left(1-\frac{r_{+}^{2}}{l^{2}}\right),
\end{aligned}
$$

where $C^{S A d S}$ blows up at $r_{0}=l / \sqrt{3}$ and $F^{S A d S}=0$ for $r_{+}=0, l$. In the limit of $b^{2} \rightarrow \infty$, these reduce to the well-known RNAdS case as follows:

$$
\begin{aligned}
& C^{R N A d S}\left(r_{+}, Q\right)=2 \pi r_{+}^{2}\left[\frac{3 r_{+}^{4}+l^{2}\left(r_{+}^{2}-Q^{2}\right)}{3 r_{+}^{4}+l^{2}\left(-r_{+}^{2}+3 Q^{2}\right)}\right], \\
& F^{R N A d S}\left(r_{+}, Q\right)=\frac{r_{+}}{4}\left(1-\frac{r_{+}^{2}}{l^{2}}+\frac{3 Q^{2}}{r_{+}^{2}}\right)-M_{e}^{R N A d S} .
\end{aligned}
$$

The global features of the Hawking temperature depending on the parameter $b$ are shown in Fig. 3. It seems to be a combination of the RN and 
Schwarzschild black holes in the AdS space for the cases of $b \geq 0.5$. Here we observe the local minimum $T_{H}=T_{0}$ at $r_{+}=r_{0}$ (the feature of the SAdS black hole), and the maximum value $T_{H}=T_{m}$ at $r_{+}=r_{m}$ and $T_{H}=0$ at $r_{+}=r_{e}$ (the feature of the RN black hole). In addition, the position $r_{+}=r_{e}$ having the extremal temperature $T_{H}=0$ is being shifted to zero when $b$ decreases from $b \rightarrow \infty$ (RNAdS) to $b=1$ (BIAdS), and then to $b=0.5$ (BIAdS). This implies that the nonlinear effects of the BI action make the extremal position close to zero, similar to the BIBTZ in three dimensions [23].

The graphs of the heat capacity depending on the parameter $b$ are shown in Fig. 4. For the SAdS case of $Q=0$, we have the typical form of heat capacity, showing the change from $-\infty$ to $\infty$ at the minimum temperature point of $r_{0}=l / \sqrt{3}$. Hence, this case has simply two phases of negative and positive heat capacities. On the other hand, for the BIAdS case of $b Q \geq 0.5$, we have the RNAdS-type heat capacities with two discontinuities at $r_{+}=r_{m}, r_{0}$ corresponding to the solution of $\left(d T_{H} / d r_{+}\right)_{Q}=0$ and zero at $r_{+}=r_{e}$ related to the solution of $T_{H}=0$. In particular, for $r_{e}<r_{+}<r_{m}$, the black hole is locally stable because of $C>0$ while for $r_{m}<r_{+}<r_{0}$, it is locally unstable $(C<0)$. For $r_{+}>r_{0}$, the black hole becomes stable because of $C>0$. As a result, we observe that $C=0$ at $r_{+}=r_{e}$, and it blows up at $r_{+}=r_{m}, r_{0}$. Based on the local stability, we introduce the stable small black hole $\left(\mathrm{SBH}_{+}\right)$with $C>0$ being in the region of $r_{e}<r_{+}<r_{m}$, the unstable intermediate black hole ( $\mathrm{IBH}_{-}$) with $C<0$ in the region of $r_{m}<r_{+}<r_{0}$, and the stable large black hole $\left(\mathrm{LBH}_{+}\right)$with $C>0$ in the region of $r_{+}>r_{0}$.

\section{Phase transitions in BIAdS black holes}

As is well-known, the free energy plays a crucial role to test the phase transition. The global features of the on-shell free energy depend on the parameter $b$. We find that the nonlinear generalization with $b Q \geq 0.5$ of the EinsteinMaxwell theory does not change the thermodynamic stability in the canonical ensemble. That is, the cases of $b=0.5,1.0$ could not make the free energy lower than the RNAdS with $b \rightarrow \infty$ in contrast to the case of the grand canonical ensemble [14], which states that the BIAdS is thermodynamically preferred (stable) over the RNAdS. This is mainly because we choose the extremal black hole as the ground state in the canonical ensemble [26]. The only change is shifting of extremal points toward the origin as $b$ decreases.

In order to investigate what kinds of the Hawking-Page phase transition are possible to occur in the BIAdS, we have to study the off-shell process of the growth of a black hole. For this purpose, we introduce the off-shell free 


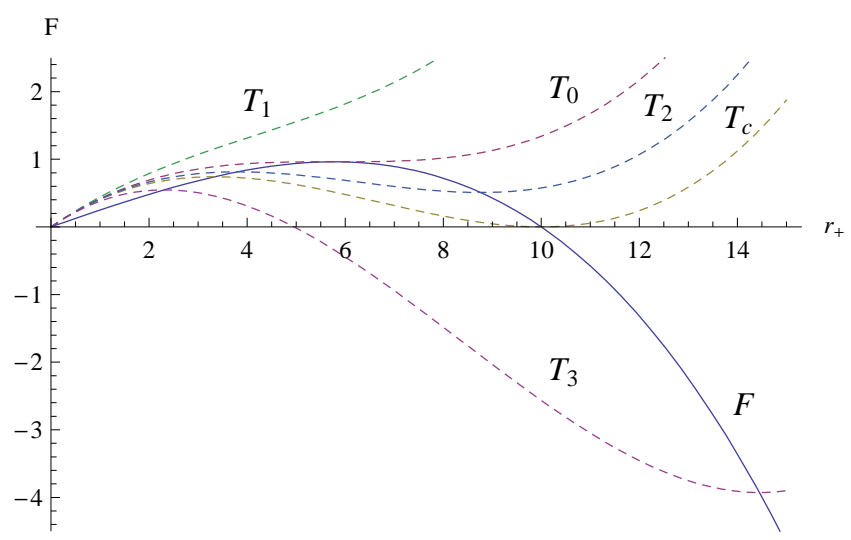

Figure 5: Phase transition for the SAdS: minimum temperature $T_{0}=0.02757$ at $r_{0}=5.774$, critical temperature $T_{c}=0.03183$ for HP2 at $r_{c}=10$. In addition, three external temperatures $T_{1}(=0.02), T_{2}(=0.03)$, and $T_{3}(=0.04)$ are introduced for the phase transition. The solid curve represents the onshell free energy $F^{S A d S}\left(r_{+}\right)$, while five dashed curves denote the off-shell free energy $F_{\text {off }}^{S A d S}\left(r_{+}, T\right)$ with five temperatures.

energy as follows

$$
F_{\text {off }}\left(r_{+}, Q, b, T\right)=M\left(r_{+}, Q, b\right)-M_{e}(Q, b)-T S_{B H}\left(r_{+}\right)
$$

with the external temperature $T$ of heat reservoir. We describe the phase transitions by considering four cases: $Q=0 \mathrm{SAdS}, b Q=0.5$ critical BIAdS, $b Q=1$ BIAdS, $b \rightarrow \infty$ RNAdS. We newly classify the Hawking-Page phase transitions according to the previous works $[27,30]$.

\section{1 $Q=0$ SAdS black hole (HP2)}

A Schwarzschild-AdS black hole (SAdS) is globally stable only when $C>0$ and $F<0$. We observe that the free energy is maximum at $r_{0}=l / \sqrt{3}$ and zero at $r_{c}=l$ as shown in Fig. 5. For $r_{+}>r_{c}$, one finds negative free energy. The temperatures correspond to minimum $T_{0}=T_{H}^{S A d S}\left(r_{0}\right)$ and $T_{c}=T_{H}^{S A d S}\left(r_{c}\right)$, respectively. Using the off-shell free energy

$$
F_{o f f}^{S A d S}=M^{S A d S}-T S_{B H}
$$

we find the phase transition. For $T=T_{3}$, the process of phase transition is shown in Fig. 5. In this case, one generally starts with thermal radiation $\left(r_{+}=0\right)$ in AdS space appearing an unstable small black hole $\left(\mathrm{SBH}_{-}\right)$at $r_{+}=$ 


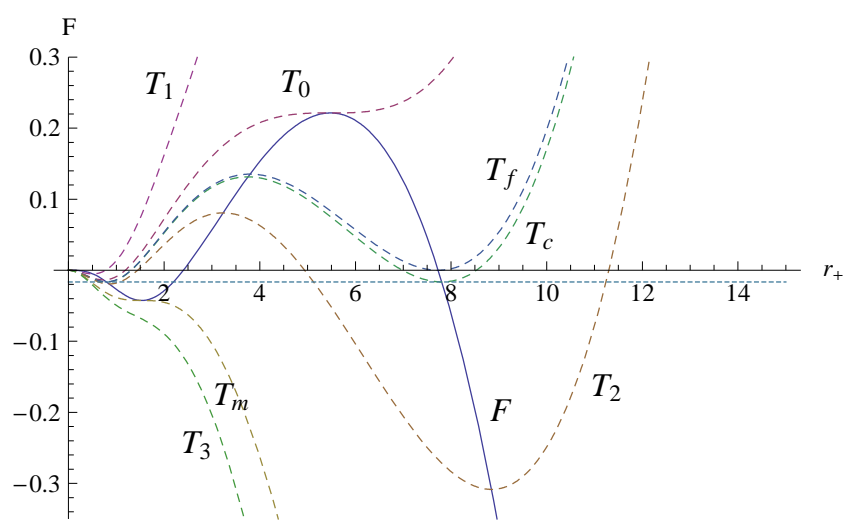

Figure 6: Phase Transition for the $b=0.5$ critical BIAdS with $Q=1$ : $T_{m}=0.03647$ at $r_{m}=1.531$, local minimum $T_{0}=0.02712$ at $r_{0}=5.478$, $T_{f}=0.02858$ at $r_{f}=7.734, T_{c}=0.02867$ at $r_{s}=0.796$ and $r_{l}=7.810$, $T_{1}(=0.02), T_{2}(=0.03), T_{3}(=0.04)$. The solid curve represents the on-shell free energy $F\left(r_{+}, Q=1\right)$, while seven dashed curves denote the off-shell free energy $F_{o f f}\left(r_{+}, Q=1, T\right)$ with seven temperatures.

$r_{u}$ (solution to $\left.F_{o n}^{S A d S}\left(r_{u}\right)=F_{\text {off }}^{S A d S}\left(r_{u}, T_{3}\right)\right)$ with negative heat capacity. Here $\mathrm{SBH}_{-}$denotes the unstable small black hole with $C<0$ and $F>0$. Then, since the heat capacity changes from negative infinity to positive infinity at $r_{+}=r_{0}$ (see Fig. 4-a), the large black hole $\left(\mathrm{LBH}_{+}\right)$with $C>0$ finally comes out as a stable object at $r_{+}=r_{s}$ (solution to $F^{S A d S}\left(r_{s}\right)=F_{o f f}^{S A d S}\left(r_{s}, T_{3}\right)$ ). Here $\mathrm{LBH}_{+}$denotes the stable large black hole with $C>0$ and $F<0$. Actually, there is a change of the dominance at the critical temperature $T_{c}$ : from thermal radiation to black hole [25]. This is called the conventional Hawking-Page phase transition (HP2): AdS $\rightarrow \mathrm{SBH}_{-} \rightarrow \mathrm{LBH}_{+}$. For the $T=T_{1}, T_{2}\left(<T_{c}\right)$ cases, the free energy of AdS space $F^{S A d S}(0)=0$ is the lowest one, while for the $T=T_{3}\left(>T_{c}\right)$ case, the lowest one is the negative free energy $F^{S A d S}\left(r_{s}\right)$ of the large black hole.

\section{$4.2 b Q=0.5$ critical BIAdS black hole (HP3)}

Here we discuss the critical BIAdS case with $b Q=0.5$ as a new phase transition. From Fig. 6, the local minimum $F=F_{\min }$ is at $r_{+}=r_{m}$, the maximum value $F=F_{\max }$ is at $r_{+}=r_{0}$, and $F=0$ at $r_{+}=r_{e}(=0)$ and $r_{f}$. The negative free energy decreases in the range of $r_{e}<r_{+}<r_{m}$, and then it increases in the region of $r_{m}<r_{+}<r_{0}$. For $r_{+}>r_{0}$, the positive free energy again decreases to zero at $r_{+}=r_{f}$, and finally becomes negative for $r_{+}>r_{f}$. On the other hand, from Fig. 4-b, we find the change of the heat capacity: 
$C=0$ at $r_{e}=0 ; C>0$ for $0<r_{+}<r_{m} ; C<0$ for $r_{m}<r_{+}<r_{0} ; C>0$ for $r_{+}>r_{0}$. Here, we define the extremal black hole $\left(\mathrm{EBH}_{0}\right)$ at $r_{+}=r_{e}$ with $T_{H}=C=F=0$.

From Fig. 6, we can propose a new type of the Hawking-Page phase transition (HP3). In this case, the critical temperature $T=T_{c}$ is derived from the condition of $F_{o f f}\left(r_{s}, 1, T_{c}\right)=F_{o f f}\left(r_{l}, 1, T_{c}\right)$, which means that the free energy of $\mathrm{SBH}_{+}$at $r_{+}=r_{s}$ is equal to free energy of $\mathrm{LBH}_{+}$at $r_{+}=r_{l}$. Here we distinguish $\mathrm{SBH}_{+}$with $\mathrm{SBH}_{-}$: the former is a stable small black hole with $C>0, F<0$, while the latter is unstable small black hole with $C<0, F>0$. Then we expect that a HP3 occurs between $\mathrm{EBH}_{0}$ and $\mathrm{LBH}_{+}$through $\mathrm{SBH}_{-}$ and $\mathrm{IBH}_{-}$for $T_{m}<T<T_{c}$. Here $\mathrm{IBH}_{-}$represents the unstable intermediate black hole with $C<0, F>0$. All of $\mathrm{SBH}_{+}, \mathrm{IBH}_{-}$, and $\mathrm{LBH}_{+}$are the saddle points as the solutions to $F=F_{\text {off }}$. Particularly, we choose $T_{2}=0.03$ for a transition temperature of the HP3. This transition consists of three processes: $\mathrm{EBH}_{0} \rightarrow \mathrm{SBH}_{+} \rightarrow \mathrm{IBH}_{-} \rightarrow \mathrm{LBH}_{+}$. We have global stabilities for $\mathrm{SBH}_{+}$and $\mathrm{LBH}_{+}$because of their free energies are negative and heat capacities are positive. However, the $\mathrm{IBH}_{-}$remains unstable. It may play a role of a mediator for HP3 as a $\mathrm{SBH}_{-}$in HP2 because of $C<0$ and $F>0$. Here we observe that the dominance of system is changed from $\mathrm{SBH}_{+}$to $\mathrm{LBH}_{+}$at $T=T_{c}$, called the critical temperature of HP3. For $T<T_{c}$, the free energy of $\mathrm{SBH}_{+}$is the lowest one, while for $T>T_{c}$, the lowest free energy is for the $\mathrm{LBH}_{+}$.

It seems appropriate to comment on $T=T_{f}$, which may be considered as the critical temperature. However, this shows the unwanted sequence of $F_{\text {off }}\left(r_{s}\right)<F_{\text {off }}\left(r_{e}\right)=F_{\text {off }}\left(r_{l}\right)(=0)<F_{\text {off }}\left(r_{i}\right)$, which implies that the transition from $\mathrm{EBH}_{0}$ to $\mathrm{LBH}_{+}$unlikely occurs because the free energy of $\mathrm{SBH}_{+}$is the lowest one. Hence, we should choose $T=T_{c}$, which shows the desired sequence of $F_{\text {off }}\left(r_{s}\right)=F_{\text {off }}\left(r_{l}\right)<F_{\text {off }}\left(r_{e}\right)(=0)<F_{\text {off }}\left(r_{i}\right)$. This case implies that a new type of the Hawking-Page phase transition from $\mathrm{EBH}_{0}$ to $\mathrm{LBH}_{+}$is possible to occur.

\section{3 $b Q=1$ BIAdS black holes (HP1)}

Now, let us discuss the BIAdS case with $b Q=1$. From Fig. 7, the local minimum $F=F_{\min }$ is at $r_{+}=r_{m}$ and the maximum value $F=F_{\max }$ is at $r_{+}=r_{0}$. The free energy is negative for $r_{e}<r_{+}<r_{m}$ and it increases in the region of $r_{m}<r_{+}<r_{0}$. For $r_{+}>r_{0}$, it decreases to zero at $r_{+}=r_{f}$ and becomes negative for $r_{+}>r_{f}$. On the other hand, from Fig. 4-c, we find the change of heat capacity: $C=0$ at $r_{+}=r_{e} \neq 0 ; C>0$ for $0<r_{+}<r_{m}$; $C<0$ for $r_{m}<r_{+}<r_{0} ; C>0$ for $r_{+}>r_{0}$. 


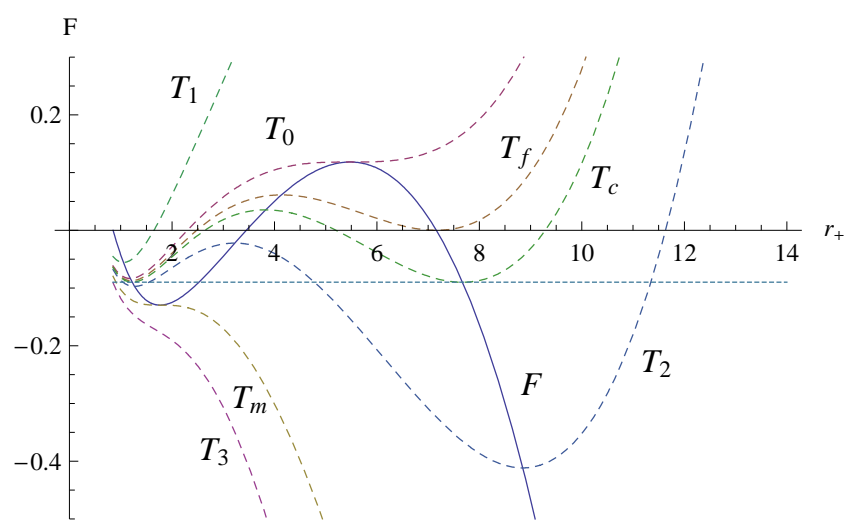

Figure 7: Phase Transition for the $b=1$ BIAdS with $Q=1: T_{m}=0.03518$ at $r_{m}=1.769$, local minimum $T_{0}=0.02712$ at $r_{0}=5.478$, temperature $T_{f}=0.02799$ at $r_{f}=7.159, T_{c}=0.0285$ at $r_{s}=1.224$ and $r_{l}=7.700$, $T_{1}(=0.02), T_{2}(=0.03), T_{3}(=0.04)$. The solid curve represents the on-shell free energy $F\left(r_{+}, Q=1\right)$, while seven dashed curves denote the off-shell free energy $F^{o f f}\left(r_{+}, Q=1, T\right)$ with seven temperatures.

From Fig. 7, we can propose another phase transition (HP1) [27, 30]. Here the off-shell free energies always start with the nonzero values at $r_{+}=r_{e}$ because of $S_{B H}\left(r_{e}\right) \neq 0$. This contrasts to the critical BIAdS. In this case, the critical temperature $T=T_{c}$ is derived from the condition of $F_{\text {off }}\left(r_{s}, 1, T_{c}\right)=$ $F_{\text {off }}\left(r_{l}, 1, T_{c}\right)$, which means that the free energy of $\mathrm{SBH}_{+}$at $r_{+}=r_{s}$ is equal to free energy of $\mathrm{LBH}_{+}$at $r_{+}=r_{l}$. Then we expect that a HP1 occurs between $\mathrm{SBH}_{+}$and $\mathrm{LBH}_{+}$through $\mathrm{IBH}_{-}$for $T_{m}<T<T_{c}$. Particularly, we choose $T_{2}=0.03$ for a transition temperature of the HP1. This transition consists of two processes: $\mathrm{SBH}_{+} \rightarrow \mathrm{IBH}_{-} \rightarrow \mathrm{LBH}_{+}$. We have global stabilities for $\mathrm{SBH}_{+}$and $\mathrm{LBH}_{+}$because of their free energies are negative and heat capacities are positive. However, the IBH_ remains an unstable mediator for HP1. Here we observe that the dominance of system is changed from $\mathrm{SBH}_{+}$ to $\mathrm{LBH}_{+}$at $T=T_{c}$, called the critical temperature of HP1. For $T<T_{c}$, the free energy of $\mathrm{SBH}_{+}$is the lowest one, while for $T>T_{c}$, the lowest free energy is for the $\mathrm{LBH}_{+}$.

\section{$4.4 b \rightarrow \infty$ BIAdS black hole (RNAdS:HP1)}

This case is similar to the $b Q=1$ BIAdS case. There is a phase transition like HP1: $\mathrm{SBH}_{+} \rightarrow \mathrm{IBH}_{-} \rightarrow \mathrm{LBH}_{+}$because of $r_{e}^{R N A d S} \neq 0$ [28]. This occurs for the case of $Q<Q_{c}$ in the canonical ensemble of the RNAdS. For the case of $Q \geq Q_{c}$, the transitions are not allowed in the RNAdS as the limiting case 
of the BIAdS [26].

\section{$5 \quad$ Summary and discussions}

In summary, we have considered the four dimensional BIAdS, which is the nonlinear generalization having the limiting case of the RNAdS. We have carefully analyzed all possible BIAdS black hole solutions, which depend on the charge $Q$ and the nonlinear parameter $b$ with the reality condition $b Q \geq 0.5$. Then, we have obtained all thermodynamic properties of the possible BIAdS comparing with those of the Reissner-Norström-AdS and Schwarzschild-AdS black holes.

Moreover, we have obtained possible Hawking-Page phase transitions in the BIAdS black holes through the analysis of the off-shell process of the growth of a black hole by introducing the off-shell free energy. The first one is $\mathrm{HP} 2$ of $\mathrm{AdS} \rightarrow \mathrm{SBH}_{-} \rightarrow \mathrm{LBH}_{+}$, which belongs to the conventional Hawking-Page phase transition in the Schwarzschild-AdS black holes. For the critical BIAdS, we have found a new phase transition (HP3) through the process of $\mathrm{EBH}_{0} \rightarrow \mathrm{SBH}_{+} \rightarrow \mathrm{IBH}_{-} \rightarrow \mathrm{LBH}_{+}$. This is similar to the phase transition in the non-rotating BTZ black hole (NBTZ) of $\mathrm{EBH}_{0} \rightarrow \mathrm{NBTZ}_{+}$ in three dimensions [40]. The difference is the number of different phases: single phase for the NBTZ and three phases for the case of the $b Q=0.5$ critical BIAdS. The phase transition for the case of the $b Q>0.5$ BIAdS is $\mathrm{HP} 1$, which shows the transition between $\mathrm{SBH}_{+}$and $\mathrm{LBH}_{+}$as $\mathrm{SBH}_{+} \rightarrow$ $\mathrm{IBH}_{-} \rightarrow \mathrm{LBH}_{+}$. For the RNAdS, which is the $b \rightarrow \infty$ BIAdS, one has the similar phase transition like HP1.

At this stage, let us discuss whether the HP1 is really possible to occur because this is the phase transition between two globally stable objects $\left(\mathrm{SBH}_{+}\right.$and $\left.\mathrm{LBH}_{+}\right)$. It was reported that the HP1 is unlikely to occur in the RNAdS [28]. This is because the maximum temperature of $T=T_{m}$ is present at the Davies' point of $r_{+}=r_{m}$ [41]. Whether the phase transition at $r_{+}=r_{m}$ is possible or not is a long-standing issue to be resolved $[42,43,44]$. The presence of maximum temperature gives rises to a stable small black hole of $\mathrm{SBH}_{+}$. If the transition between $\mathrm{SBH}_{+}$and $\mathrm{IBH}_{-}$is not possible, two transitions of HP3 and HP1 except HP2 are unlikely possible to occur. In this work, we have discussed all phase transitions by assuming that the phase transition between $\mathrm{SBH}_{+}$and $\mathrm{IBH}_{-}$is likely to occur.

Now we ask whether the case of $b Q=0.5$ BIAdS is really a critical point for the transition to the Schwarzschild-AdS black holes. As was pointed out previously, it is not the case because the BI action (BIAdS) is a nonlinear 
generalization of Maxwell theory (RNAdS). The key point of the RNAdS is that it has an extremal point of zero temperature when compared with the SAdS. Hence, we insist that discussing the transition to the SAdS is irrelevant to the BIAdS.

Finally, we discuss the implications of our results to the string theory. According to the AdS/CFT correspondence, the Hawking-Page phase transition of HP2 corresponds to the confining-deconfining phase transition on the CFT side [45]. Because the BI action is related to the string theory when choosing $b=1 / 2 \pi \alpha^{\prime}$, the phase transitions of HP3 and HP1 could be applied to explore the presumed transitions in the CFT.

\section{Acknowledgments}

Y. S. Myung was supported by the Korea Research Foundation (KRF-2006311-C00249) funded by the Korea Government (MOEHRD). Y.-W. Kim was supported by the Korea Research Foundation Grant funded by Korea Government (MOEHRD): KRF-2007-359-C00007. Y.-J. Park was supported by the Korea Science and Engineering Foundation (KOSEF) grant funded by the Korea government (MOST) (R01-2007-000-20062-0).

\section{Appendix: Free energy from the Euclidean ac- tion approach}

In the appendix, we derive the free energy in Eq. (14) from the Euclidean action approach $[26,14]$. In general, there are two approaches: the grand canonical thermodynamic ensemble for the fixed-potential and canonical thermodynamic ensemble for the fixed-charge. For the AdS space, the background consists of both charged and uncharged quanta free to fluctuate in the presence of fixed-potential $\Phi$. In the case of grand canonical ensemble at fixedtemperature and fixed-potential, the relevant thermodynamic potential is the Gibbs free energy defined by $W=M-T_{H} S_{B H}-\Phi Q$. The proper free energy for the fixed-potential was derived in Ref. [14].

On the other hand, for the fixed-charge $Q$, the AdS space with a fixedcharge is not a solution to the BIAdS equations. In this case, in order to keep the fixed-charge, we use the extremal black hole with the mass $M_{e}$ as the background and retain only uncharged (neutral) quanta in the heat 
reservoir. Here, the relevant quantity is the Helmholtz free energy defined by $F=E-T_{H} S_{B H}$ with $E=M-M_{e}$.

In this appendix, we derive the desired free energy for the fixed-charge from the Euclidean action approach. In order to compute the free energy for the case of a fixed-charge $Q$ canonical ensemble, let us consider the Euclidean action

$$
I_{E}=I_{b}+I_{G H Y}+I_{s}-I_{c}-I_{e}
$$

Here $I_{b}$ is the Euclidean version (bulk term) of $S_{0}$ in Eq. (1), while the remaining terms are all boundary terms. These boundary terms except $I_{e}$ fall into two classes: boundary terms $I_{G H Y}$ and $I_{s}$, which are required to get the correct boundary value problem, belong to type I, while counter-term $I_{c}$, which is required to get the correct variational principle, belongs to type II.

Among boundary terms, $I_{G H Y}$ is the Gibbons-Hawking-York term

$$
I_{G H Y}=\int_{\partial M} d^{3} x \sqrt{h} K
$$

where $K$ is the trace of extrinsic curvature. When the space is asymptotically AdS, $I_{G H Y}$ gives a vanishing contribution [26].

Upon variation of the gauge field, the boundary terms from the gauge field will vanish if one keeps the potential $A_{t}(\infty)=\Phi$ fixed. However, if we wish to keep the charge fixed, we must add a boundary surface term

$$
I_{s}=-\frac{1}{4 \pi G} \int_{\partial M} d^{3} x \sqrt{h}\left(\frac{F^{\mu \nu}}{\sqrt{1+2 F / b^{2}}}\right) n_{\mu} A_{\nu},
$$

where $h_{i j}$ is the induced metric on the boundary at $r=r_{B}$ and $n_{\mu}$ is a radial unit vector pointing outwards.

The counter term $I_{c}=I_{A d S}=-(\Lambda / 8 \pi G) \int_{0}^{r_{B}} d^{4} x \sqrt{g}$ is the Euclidean action of the pure AdS space, which is necessary to regularize the divergence on the boundary at infinity. This is the standard AdS counter term.

The last term $I_{e}$ is necessary to have the action for the fixed-charge ensemble, using the extremal black hole as the background. In order to compute the action, we evaluate $I_{b}+I_{G H Y}+I_{s}-I_{c}$ for a black hole of mass $M>M_{e}$ and then subtract the contribution from the extremal background. This is because the ground state of the BIAdS for the fixed-charge is not the AdS space but the extremal black hole defined by the thermal condition of $T_{H}=0, C=0, F=0$. The extremal black hole is considered as a small, stable remnant against evaporating process via the Hawking radiation. Hence, we may consider $I_{c}\left(I_{e}\right)$ the IR (UV) counter-terms to have a proper, thermodynamic system. 
Now, we are ready to obtain the proper Euclidean action (1). First, let us calculate the bulk term. Considering the equation of motion for $g_{\mu \nu}$

$$
R_{\mu \nu}-g_{\mu \nu} \Lambda=\frac{1}{2} g_{\mu \nu}\left(-\mathcal{L}(F)+\frac{\partial \mathcal{L}}{\partial g^{\rho \sigma}} g^{\rho \sigma}\right)-\frac{\partial \mathcal{L}}{\partial g^{\mu \nu}}
$$

the bulk term can be rewritten as

$$
I_{b}=-\frac{1}{16 \pi G} \int_{M} d^{4} x \sqrt{g}\left(2 \Lambda-\mathcal{L}(F)-\frac{2 F^{2}}{\sqrt{1+F^{2}}}\right) .
$$

After substituting the electric field $F_{r \tau}$ into $I_{b}$ with

$$
F_{r \tau}=i F_{r t}=\frac{-i Q}{\sqrt{r^{4}+\frac{Q^{2}}{b^{2}}}}
$$

it could be integrated to give the form

$$
I_{b}=\frac{\omega \beta}{16 \pi G}\left[\frac{2}{l^{2}}\left(r_{B}^{3}-r_{+}^{3}\right)+\frac{4 b^{2}}{3}\left(r_{B}^{3}-r_{+}^{3}\right)-4 b \int_{r_{+}}^{r_{B}} \sqrt{r^{4}+Q^{2}}\right] .
$$

Here $\omega$ is the volume of the two-sphere and $\beta$ is the inverse temperature.

Second, the surface term $I_{s}$ is computed to have

$$
I_{s}=\frac{\omega \beta}{16 \pi G} \frac{Q^{2}}{r_{+}} F\left(\frac{1}{4}, \frac{1}{2}, \frac{5}{4},-\frac{Q^{2}}{b^{2} r_{+}^{4}}\right) \equiv \frac{\omega \beta}{16 \pi G} Q \Phi,
$$

where $\Phi$ is the potential at the horizon.

Third, the counter term is calculated to be

$$
I_{c}=-\frac{\omega \beta_{0}}{16 \pi G} \int_{0}^{r_{B}}\left[\frac{6 r^{2}}{l^{2}}\right] d r .
$$

Here $\beta_{0}$ is the time period for the AdS space. It has to be rescaled to match the period $\beta$ as

$$
f(r) \beta^{2}=\left(1+\frac{r^{2}}{l^{2}}\right) \beta_{0}^{2}
$$

Then, this gives us

$$
\begin{aligned}
\beta_{0}=\beta & {\left[1-\frac{M l^{2}}{r^{3}}+\frac{2 b^{2} l^{2}}{3}\left(1-\sqrt{1+\frac{Q^{2}}{b^{2} r^{4}}}\right)\right.} \\
& \left.+\frac{2 Q^{2} l^{2}}{3 r^{4}} \mathcal{F}\left(\frac{1}{4}, \frac{1}{2}, \frac{5}{4},-\frac{Q^{2}}{b^{2} r^{4}}\right)-\frac{3 l^{4}}{8 r^{4}}\right]\left.\right|_{r=r_{B}}
\end{aligned}
$$


up to $r^{-4}$ after some approximations.

Finally, the background term of extremal black hole takes the form

$$
I_{e}=\frac{\omega \beta M_{e}}{4 \pi G}
$$

Here $M_{e}$ is given by Eq. (9).

By carefully matching the geometries of the AdS space and the black hole in the asymptotic region and sending $r_{B}$ to infinity, the desired result for the Euclidean-Born-Infeld action can be obtained as

$$
\begin{aligned}
I_{E}=\frac{\omega \beta}{16 \pi G}[ & r_{+}-\frac{r_{+}^{3}}{l^{2}}-\frac{2 b^{2} r_{+}^{3}}{3}\left(1-\sqrt{1+\frac{Q^{2}}{b^{2} r_{+}^{4}}}\right) \\
& \left.+\frac{8 Q^{2}}{3 r_{+}} F\left(\frac{1}{4}, \frac{1}{2}, \frac{5}{4},-\frac{Q^{2}}{b^{2} r_{+}^{4}}\right)-4 M_{e}\right] .
\end{aligned}
$$

In deriving this expression, we have used the condition of $f\left(r_{+}\right)=0$. As a check, we recover that in the limit of $b \rightarrow \infty$, the above action reduces to the Euclidean action for the RNAdS case [26] as

$$
I_{R N A d S}=\frac{\omega \beta}{16 \pi G}\left(r_{+}-\frac{r_{+}^{3}}{l^{2}}+\frac{3 Q^{2}}{r_{+}}-\frac{4}{3} r_{e}-\frac{8}{3} \frac{Q^{2}}{r_{e}^{2}}\right) .
$$

Since the free energy is given by $F=I_{E} / \beta$ with $\omega=4 \pi, G=1, I_{E}$ leads to the free energy (14) in the canonical ensemble. Hence we exactly derive

the free energy which was used to discuss the whole phase transitions in the text.

\section{References}

[1] M. Born and L. Infeld, Proc. R. Soc. London A 144, 425 (1934).

[2] E. S. Fradkin and A. A. Tseytlin, Phys. Lett. B 163, 123 (1985).

[3] A.A. Tseytlin, Nucl. Phys. B 276, 391 (1986) [Erratum-ibid. B 291, 876 (1987)];

[4] R. G. Leigh, Mod. Phys. Lett. A 4, 2767 (1989).

[5] A. Garcia, H. Salazar and J.F. Plebanski, Nuovo. Cim 84, 65 (1984).

[6] M. Demianski, Found. Phys. 16, 187 (1986). 
[7] D.L. Wiltshire, Phys. Rev. D 38, 2445 (1988).

[8] D. A. Rasheed, [arXiv:hep-th/9702087].

[9] T. Tamaki and T. Torii, Phys. Rev. D 62, 061501 (2000).

[10] G. W. Gibbons and C. A. R. Herdeiro, Class. Quant. Grav. 18, 1677 (2001).

[11] N. Breton, Phys. Rev. D 67, 124004 (2003).

[12] M. Aiello, R. Ferraro and G. Giribet, Exact solutions of Lovelock-BornInfeld black holes, Phys. Rev. D 70, 104014 (2004).

[13] T. K. Dey, Phys. Lett. B 595, 484 (2004).

[14] S. Fernando, Phys. Rev. D 74, 104032 (2006).

[15] A. Sheykhi, Phys. Lett. B 662, 7 (2008).

[16] S. Carlip, J. Korean Phys. Soc. 28, S447 (1995) [arXiv:gr-qc/9503024].

[17] R. Mann, Lower dimensional black holes: Inside and out, [arXiv:grqc/9501038].

[18] V. Frolov, S. Hendy and A. L. Larsen, Nucl. Phys. B 468, 336 (1996).

[19] M. Banados, C. Teitelboim and J. Zanelli, Phys. Rev. Lett. 69, 1849 (1992).

[20] M. Banados, M. Henneaux, C. Teitelboim and J. Zanelli, Phys. Rev. D 48, 1506 (1993).

[21] C. Martinez, C. Teitelboim and J. Zanelli, Phys. Rev. D 61, 104013 (2000).

[22] M. Cataldo and A. Carcia, Phys. Lett. B 456, 28 (1999).

[23] Y. S. Myung, Y.-W. Kim and Y.-J. Park, Phys. Rev. D 78, 044020 (2008).

[24] R. Yamazaki and D. Ida, Phys. Rev. D 64, 024009 (2001).

[25] S. W. Hawking and D. N. Page, Commun. Math. Phys. 87, 577 (1983).

[26] A. Chamblin, R. Emparan, C. V. Johnson and R. C. Myers, Phys. Rev. D 60, 064018 (1999). 
[27] T. K. Dey, S. Mukherji, S. Mukhopadhyay and S. Sarkar, JHEP 0709, 026 (2007).

[28] Y. S. Myung, Mod. Phys. Lett. A 23, 667 (2008).

[29] Y. M. Cho and I. P. Neupane, Phys. Rev. D 66, 024044 (2002).

[30] T. K. Dey, S. Mukherji, S. Mukhopadhyay and S. Sarkar, JHEP 0704, $014(2007)$.

[31] S. Nojiri and S. D. Odintsov, Phys. Lett. B 521, 87 (2001) [Erratumibid. B 542, 301 (2002)].

[32] M. Cvetic, S. Nojiri and S. D. Odintsov, Nucl. Phys. B 628, 295 (2002).

[33] S. Fernando and D. Krug, Gen. Relativ. Gravit. 35, 129 (2003).

[34] R. G. Cai, D. W. Pang and A. Wang, Phys. Rev. D 70, 124034 (2004).

[35] O. Miskovic and R. Olea, Phys. Rev. D 77, 124048 (2008).

[36] D. Grumiller, W. Kummer, and D. V. Vassilevich, Phys. Rept. 369, 327 (2002).

[37] D. Grumiller and R. McNees, J. High Energy Phys. 0704, 074 (2007).

[38] Y. S. Myung, Y. W. Kim and Y. J. Park, Mod. Phys. Lett. A 23, 91 (2008).

[39] M. Abramowitz and I.A. Stegun, Handbook of Mathematical Functions, Dover, 1972.

[40] Y. S. Myung, Phys. Lett. B 638, 515 (2006).

[41] P. C. W. Davies, Proc. R. Soc. Lond. A 353, 499 (1977).

[42] D. Pavon, Phys. Rev. D 43, 2495 (1991).

[43] J. Jing and Q. Pan, Phys. Lett. B 660, 13 (2008).

[44] Y. S. Myung, Y.-W. Kim and Y.-J. Park, Phys. Lett. B 663, 342 (2008).

[45] E. Witten, Adv. Theor. Math. Phys. 2, 505 (1998).

[46] G. W. Gibbons, M. J. Perry and C. N. Pope, Class. Quant. Grav. 22 1503 (2005). 